\title{
Immunogenomics: recent discoveries
}

\author{
Mayank Gupta ${ }^{1}$, Nikhil Gupta ${ }^{1}$, Soham Trivedi' ${ }^{2}$, Prasad Patil ${ }^{3}$, Girish Gupta ${ }^{4}$, \\ Vamsi Krishna $K^{5}$, Hipal Gaudani ${ }^{2}$ and Gomase V.S. ${ }^{6}$ \\ ${ }^{1}$ Sindhu Mahavidyalaya Panchpaoli Nagpur \\ ${ }^{2}$ V.V.P. Engineering College, Rajkot, Gujrat \\ ${ }^{3}$ Dr. D.Y. Patil ACS College, Pimpri, Pune \\ ${ }^{4}$ S.D.S.M. College Palghar, Mumbai \\ ${ }^{5}$ Rai foundations College CBD Belapur Navi Mumbai \\ ${ }^{6}$ School of Technology, S.R.T.M. University, Sub-Centre, Latur, 413512, India
}

\begin{abstract}
Immunogenomics is a new research field, which addresses the interface between host and (pathogen) proteome, bridging informatics technology, genomics, proteomics, immunology and clinical medicine. The switch from the paradigm of solely hypothesis-driven immunological research to a more interactive and flexible relationship between classical research and a new discovery-driven approach is termed immunogenomics. This review will focus on the immunogenomics and recent discoveries in Immunogenomics, which are leading to insights into novel immunomodulatory therapies. The research regarding the different aspects of immunogenomics; rather than providing a comprehensive review, we will highlight various factors that influence immunogenomics and how modulation of the immune response may be incorporated into future trends.

Keywords- Immunogenomics, Genetic regulation, MS, GC, NMR, microarray
\end{abstract}

\section{Introduction}

Immunogenomics a new research field used to describe the switch from the paradigm of solely hypothesis-driven immunological research to a more interactive and flexible relationship between classical research and a discovery-driven approach [1]. At least four major aspects of immunogenomics could be identified. Immunogenomics covers:

1. The convergence of distinct elements of the biological immune system into a network of information regarding the localization, gene structure and expression profile of various players of the innate and/or acquired immune response;

2. Genetic regulation of physiological immune functions by inherited or epigenetic processes such as immunoglobulin or $\mathrm{T}$ cell receptor gene rearrangement, somatic hyper-mutation, immune selection in primary and peripheral immune tissues, antigen processing and presentation by major histocompatibility molecules, cytotoxic interactions, etc.

3. Genetic changes in immune systems in pathological conditions such as oncohematological diseases, allergy, immune deficiencies, infections, chronic inflammation, autoimmunity and cancer.

4. Suggest personalized approaches to immune therapy by predicting successful treatment or deleterious side effects and also, helping in the selection of treatments appropriate for individual patients.

This new research era will start when such alterations could be easily collected during clinical trials through inexpensive high-throughput methods for detection of genomic variation or for expression profiling that could be applied to large patient populations [2-4]. With this strategy it will then be possible to immune phenotype individuals according to their genetic make-up and the epigenetic adaptations of their immune system, however, the kinetics of individual immune responses, the network in which they will operate and their vulnerability in sickness or in health may be predicted for each individual [5]. The immunogenomics approach stands on three foundations, these are as:

a. Revolutionary expansion of genome knowledge that is now available in giant computer databanks [6, 7]

b. Robust nanotechnology such as microarray chips and similar tools that allow realtime measurement of gene variants and gene expression and

c. Availability of improving software principles in immune bioinformatics that could generate data-mining tools for an efficient interpretation of otherwise unmanageable biological information [8].

\section{Background and history}

Genomic and proteomics studies provide scientists with methods to quickly analyse genes and their products en masse. The first highthroughput techniques to be developed were sequencing methods. Genomics is now shifting to the study of gene expression and function. In the past 5-10 years genomics, proteomics and highthroughput microarray technologies have fundamentally changed our ability to study the molecular basis of cells and tissues in health and diseases, giving a new comprehensive view [9]. In 1994, a complementary DNA (cDNA) sequencing project that aimed to predict the primary structures of unknown human proteins 
was pioneered. In parallel, many groups have made a tremendous amount of effort to develop various resources for functional human genomics. In this context, to demonstrate the power of functional genomic approaches in practice, these cDNA have been applied for a comprehensive understanding of the immune system, which was termed 'immunogenomics'[10]. To better understand immune systems function and regulation, transcriptome projects (e.g., Functional Annotation of the Mouse), which focus on sequencing full-length transcripts from multiple tissue sources, ideally included specific immune cells (e.g., B cells, T cell, macrophages, dendritic cells) at various states of development, in activated and unactivated states and in different disease contexts were started[11].

\section{Immunogenomics and mass spectrometry}

Mass spectrometry has been widely used in the field of immunogenomics research and one of the applications of mass spectrometry was in the global analysis of porcine alveolar macrophage proteins through two-dimensional electrophoresis and mass spectrometry. Representative set of the major proteins was subjected to MALDI-TOF analysis and 106 proteins were identified on the basis to their similarity with the human homologous proteins. Large number of cytoskeletal and metabolic proteins were found as well as some proteins related to cell mobility and immunological functions. Finally, other proteins implicated in the cell signalling process, transport or apoptosis were also identified giving a wide overview of the porcine AM protein map. However, results presented provide a useful tool for future studies on AM biology, as well as for identifying the proteins implicated in the immune response or physiological changes that could be correlated with porcine diseases [12].

\section{Immunogenomics and microarray}

DNA Chip or Microarray technology is an important tool in the field of immunogenomics and is being used for the identification of a gene expression signature of infection during transcriptional profiling of peripheral blood mononuclear cells (PBMC) from tuberculosisinfected and healthy control cattle. Microarray analysis of messenger RNA abundance is used to investigate differential gene expression of PBMC from six cattle infected with Mycobacterium bovis and six uninfected controls. New targeted immunospecific cDNA microarray platform representing 1391 genes was used with a reference experimental design. Real-time quantitative reverse transcription PCR in eight animals per treatment group validated the microarray results. Thus it suggests that gene expression profiling may form the basis of novel immunological tests to enhance diagnosis of $M$. bovis infection in cattle [13]. Comparative study in vivo and in vitro functional capacities of leukocytes from non-parturient and periparturient dairy cows have provided substantial evidence that systemic and local mammary immune defenses are deficient around parturition. Research evidence has lead to the reasonable hypothesis that immune deficiency underlies the heightened mastitis susceptibility of periparturient cows. cDNA microarray technology has been usedto explore bovine leukocyte RNA for global gene expression changes occurring around parturition. Sequence alignment BLASTN analysis of these genes revealed only one that can be considered a classical immune response gene. All other repressed genes were either unknown or putatively identified as encoding key proteins involved in normal growth and metabolism of cells. Also, cDNA microarray technology plays critical roles in finding periparturient immunosuppression and mastitis susceptibility [14]. Recent scientific advances in tumor immunology have fostered the clinical implementation of different immunotherapy modalities. The complexity of the immune system network and the multidimensionality of tumorhost interactions, the comprehension of tumor immunology might greatly benefit from highthroughput DNA array analysis that can portray the molecular kinetics of immune response on a genome-wide scale, thus accelerating the accumulation of knowledge and ultimately catalyzing the development of new hypotheses in cell biology. The applications of DNA array technology in tumor immunology studies has already provided investigators with novel data and intriguing hypotheses on the cascade of molecular events leading to an effective immune response against cancer [15]. Current research trends, the DNA-array approach will be extremely prolific in identifying and characterizing biological phenomena and will provide, as a consequence, biologically targeted therapies. This will be particularly the case for cancer immunotherapy [16].

\section{Immunogenomics and RT-PCR}

Real time-PCR (RT-PCR) researches have been used in the comparative gnomic analysis of the genomes. RT-PCR was performed.The average nucleotide sequence divergence of intronic regions between the quail and chicken was $16.2 \%$ for 13 genes, which is similar to the average divergences in other chromosomal intronic regions (14-17\%) and in the mitochondrial sequence (14.3\%). This sequence diversity, chicken microsatellite primers did not generally allow successful PCR amplification in the quail genome [17]. The intronic regions have supposedly undergone neutral selection, although they are surrounded by the exonic regions with a high degree of nucleotide and 
amino acid diversities that have been maintained under positive selection. Based on the average of $16.2 \%$ nucleotide divergence in the intronic regions, the divergence time between the quail and the chicken $(\mathrm{d}=0.162)$ was estimated to be $81 \pm 1.9$ million years ago [18-20]. To the current scenario, this divergence time is the first estimation based on a genomic sequence comparison between these two species. Thus, such comparative genomic studies among avian species give us more information about the immunological, genetic and evolutionary differences that exist between avian genomes [21-24]. Intercellular adhesion molecule 1 (ICAM1 ) is a cell surface glycoprotein member of the immunoglobulin superfamily and is actively involved in immune and inflammatory responses. New polymerase chain reaction-sequencespecific primers (PCR-SSP) method for rapid and simultaneous genotyping of ICAM-1 G241R and K469E polymorphisms is being introduced. PCRSSP-based genotyping protocols are more convenient and cost-effective to do, it could therefore offer a valuable tool for assessment of ICAM-1 polymorphisms to which more confirmatory studies are needed [25].

\section{Immunogenomics and gas chromatography}

Gas chromatography methodology is widely used in the field of immunogenomics and is used during the study of individual $\mathrm{MHC}$ and background genes in the urinary odors of mice. Comparison between urinary volatile profiles of two MHC haplotypes ( $\mathrm{H} 2(\mathrm{~b})$ and $\mathrm{H} 2(\mathrm{k}))$ and their heterozygous cross (H2(b) x H2(k)) for two different background strains (C57BL/6J and $\mathrm{BALB} / \mathrm{c}$ ) using gas chromatography/mass spectrometry (GC/MS) was done. $\mathrm{MHC}$ and background genes substantially influence the volatile profile. Of 148 compounds screened, 108 of them significantly differ between the six genotypes. Surprisingly, for numerous compounds, their MHC associations are moderated by background genes. Also, effects of Major histocompatibility complex (MHC) variation on concentrations of metabolites and the interaction between $\mathrm{MHC}$ and other genetic variation implies the existence of unknown processes by which variation in $\mathrm{MHC}$ genes gives rise to variation in volatile molecules in body fluids [26]. Major histocompatibility complex $(\mathrm{MHC})$ is the most polymorphic genetic system known in vertebrates. Also been suggested, MHC genes influence social behavior and reproductive phenomena. However, work in this field has only been carried out in rodents and humans. To explore the relationship between individual odor profiles and MHC alleles, study was carried out on ring-tailed lemurs (Lemur catta). Individual odor profiles from tail and scent gland samples were generated for six males using gas chromatography mass spectrometry (GC-
MS).The GC-MS analyses demonstrated a difference between profiles obtained from tail and scent gland samples. The analyses suggest a relationship between $\mathrm{MHC}$ and concentrations of volatile compounds [27].

\section{Immunogenomics and NMR}

With recent advances Immunogenomics and NMR methodology, it now appears that NMR can be used at an unprecedented level of sophistication to obtain new insights into the solution structure and dynamics of the antibody combining site, both free and in its complex with antigen. Most promising in this regard is the Fv fragment (molecular weight approximately 25 kD), which can be produced by genetic engineering in a form suitable for NMR studies. NMR can also provide information on the conformational preferences of immunogenic peptides and can be used to probe the conformation and dynamics of peptides (appropriately labeled with $13 \mathrm{C}$ or $15 \mathrm{~N}$ ) bound to the Fab fragment (molecular weight approximately $50 \mathrm{kD}$ ) of antipeptide antibodies [28].

\section{Current research scenario}

In Current research scenario, xenotransplantation is one of the most challenging research areas in the field of immunogenomics and such research on somatic cloning of pigs expressing various immunmodulatory transgenes is done. The hyperacute rejection response (HAR), which was the first hurdle in porcine-to-human xenotransplantation, can now be overcome in a clinically relevant manner by expression of human complement regulatory proteins (DAF, hCD59, CD46) in transgenic pigs. Current goal of research is the generation and characterization of improved lines of multi-transgenic pigs targeting the AVR and specifically this coagulation disorder. Transgenic pigs expressing constructs for the human thrombomodulin (hTM) are produced. Porcine kidneys from these multitransgenic pigs are used in in vitro perfusion assays with human blood. New generation of multi-transgenic pigs with high expression of the various molecules will significantly improve longterm survival of porcine xenografts and will be a major step forward in the clinical application of xenotransplantation [29]. The Genomics of Salmonella enterica serovar Typhimurium invasion in pig Jejunum is being studied. Research is being carried on Salmonella enterica serovar Typhimurium species to get information on in vivo interactions during actual invasion. The investigation is carried out on early interaction of Salmonella with intact pig small intestinal mucosa using the pig small intestinal segment perfusion (SISP) model. The genes involved are part of the innate immune system, 
and regulate the inflammatory response. The most striking conclusion is the limited number of differentially expressed genes in intact mucosa compared to in vitro results. This suggests that $S$. typhimurium is adapted to evade in vivo host responses by down regulating the local inflammatory response [30]. Transcriptomic approach for the study of modification of the porcine immature dendritic cell transcriptome upon interaction with Pseudorabies virus has been introduced. Pseudorabies virus (PrV) is a well-studied pathogen and a good model to study the mechanisms of development of innate and adaptive immune response in the pig species. For identification of the genes involved in development of innate and adaptive immune response during the course of a viral infection a transcriptomic approach in which modifications of cellular transcriptome and expression of all PrV genes were simultaneously analysed at several times post-infection. The analysis of the transcriptomic profiles is presented [31]. Immunogenomic study has a major impact on the MHC molecules study and such research is on $B A C$ transgenic mouse model to analyze the role of the bovine FcRn in IgG and albumin metabolism. The $\mathrm{MHC}$ class I related Fc receptor for IgG (FcRn) protects IgG from intracellular catabolic degradation, plays important roles in IgG transcytosis in several mucosal layers and is involved in the maternal immune transport. Most recently, it has been shown that FcRn binds albumin and protects it from degradation just as it does IgG. BAC transgenic mice overexpressing the bovine FcRn heavy chain is developed and it is detected that bovine FcRn is expressed in endothelial cells too and Pharmacokinetic studies showed that IgG with enhanced binding to FcRn has increased serum persistence in cattle, and thus FcRn can effectively protect IgG from degradation. These studies indicate that the bovine FcRn heavy chain is indeed expressed in the mouse endothelial cells and form a functional receptor that protects both IgG and albumin [32]. DNA typing is being used to study the haplotypes and of swine class II major histocompatibility complex polymorphisms have been used to identify porcine haplotypes. In humans and swine (who have $85 \%$ homology in $\mathrm{MHC}$ class II), exon 2 of $\mathrm{MHC}$ class II genes encodes the first domain of the beta-chain and this domain is in direct contact with the processed peptide and/or the Tcell receptor during antigen presentation. The research includes the use of DNA of divergent breeds. Different approaches like primer targeting, PCR amplifications is used to show that Specific polymorphisms in these class II genes leads to an increase in susceptibility to diseases such as pneumoconiosis, autoimmune diseases, and allo/xeno-graft rejection [33].

\section{Discussion and Conclusion}

Progress in deciphering immune regulatory networks and immune systems will require the cooperative efforts of immunogeneticists, immunologists, molecular biologists and bioinformaticians. Although primary sequence analysis remains useful for annotation of new transcripts it is less useful for identifying novel functions of known transcripts in a new context i.e. protein interaction network or pathway. The most efficient research approach to mine useful information from the vast a priori knowledge contained in biological databases and the scientific literature, is to use a combination of computational and expert-driven knowledge discovery strategies. Also, to interpret the large amount of data, extensive computational development is required [11]. In the future research scenario, we will see the study of biological networks dominating the scene in Physiology. The great accumulation of genomics information will be used in computer programs to simulate biologic processes. In medicinal sciences, scientific results and applied biotechnologies arising from genomics will be used for effective prediction of diseases and risk associated with drugs. Preventive medicine and medical therapy will be personalized. Widespread applications of genomics for personalized medicine will require associations of gene expression pattern with diagnoses, treatment and clinical data. This will help in the discovery and development of drugs [9]. T-cell-epitope-mapping applications have now expanded to include reengineering of protein therapeutics i.e. a process now called deimmunization, as well as the fields of autoimmunity, transplantation endocrinology, allergy, and diagnostics research. Research employing T-cell-epitope mapping falls within the realm of immunogenomics, a new field that addresses the interface between host and (pathogen) proteome, bridging informatics, genomics, proteomics, immunology and clinical medicine [35]. Gene expression profiling may form the basis of novel immunological tests [13]. Comparative genomics studies among species give us more information about the genetic, immunological, and evolutionary differences that exist between their genomes [17]. New research insights into the solution structure and dynamics of the antibody combining site, both free and in its complex with antigen now can be performed. Genomics and integrated systems biology are huge tasks and no single lab can pursue this alone and is a great need for more information and better understanding of genomics before complete public acceptance.

\section{References}

[1] Marincola F.M., (2003) J Trans/ Med.1:1.

[2] Wang E., Panelli M.C., Marincola F.M.,( 2003) Princ Pract Oncol.17:1-16.

[3] Jin P., Wang E., (2003) J Trans/ Med.1:8. 
[4] Wang E., Adams S., (2001) ASHI Quarterly.24:45-48.

[5] Poon T.C., Johnson P.J., (2001) Clin Chim Acta.313:231-239.

[6] Zeeberg B.R., Feng W., Wang G., Wang M.D., Fojo A.T., Sunshine M., et al. (2003) Genome Biol.4:R28.

[7] Weinstein J.N., Scherf U., Lee J.K., Nishizuka S., Gwadry F., Bussey A.K., et al. (2002) Cytometry.47:46-49.

[8] Marincola F.M., Wang E., Atkins M.B., (2004) Exp Opin Biol Ther.4:1-7.

[9] Hocquette J.F.J, (2005 Jun) Physiol Pharmacol.56 Suppl 3:37-70INRA.

[10] Ohara O., (2009) FEBS Lett.583(11):16627.

[11] Schönbach C., ( 2003) Novartis.Found Symp.254:177-88

[12] Pérez E., Ramirez-Boo M., Garrido J.J., Moreno A. (2007) Developmental \& Comparative Immunology, 31 (12), 1220-1232.

[13] Meade K.G., Gormley E., O'Farrelly C., Park S.D., Costello E., Keane J., Zhao Y., MacHugh D.E. (2008) BMC Genomics. 9:447.

[14] Burton J.L., Madsen S.A., Yao J., Sipkovsky S.S., Coussens P.M. (2001) Acta Vet Scand. 42(3):407-24.

[15] Wang E, Marincola F.M., (2000) Immunol Today, 21: 619-23.

[16] Inoue-Murayama M., Kayang B. B., Kimura K., Ide H., Nomura A., Takahashi H., Nagamine Y., Takeda T., Hanada H., Tatsuda K., et al (2001) Anim. Genet. 32:7.

[17] Takashi Shiina, Sayoko Shimizu, Kazuyoshi Hosomichi, Sakae Kohara, Seiki Watanabe, Kei Hanzawa, Stephan Beck, Jerzy K. Kulski and Hidetoshi Inoko (2004) The Journal of Immunology, 172: 6751-6763.

[18] Kaufman J., Milne S., Gobel T. W., Walker B. A., Jacob J. P., Auffray C., Zoorob R., Beck S. (1999) Nature 401:923.

[19] Shiina T., C. Shimizu A., Oka Y., Teraoka T., Imanishi T., Gojobori K., Hanzawa S., Watanabe H., Inoko. (1999) Immunogenetics 49:384.

[20] Shiina T., Ando A., Imanishi T., Kawata H., Hanzawa K., Gojobori T., Inoko H., Watanabe S. (1995) Immunogenetics 42:213.
[21] Shiina T., Oka A., Imanishi T., Hanzawa K., Gojobori T., Watanabe S., Inoko $\mathrm{H}$. (1999) Immunogenetics 49:456.

[22] Hess C. M., Gasper J., Hoekstra H. E., Hill C. E., Edwards S. V. (2000) Genome Res. 10:613.

[23] Edwards S. V., Gasper J., Garrigan D., Martindale D., Koop B. F. (2000) Mol. Biol. Evol. 17:1384.

[24] Gasper J. S., Shiina T., Inoko H., Edwards S. V. (2001) Genomics 75:26.

[25] Tajik N., Salari F., Hajilooi M., Amoli M., Salekmoghaddam A., (2007) Tissue Antigens.69(4):338-41.

[26] Willse A., Kwak J., Yamazaki K., Preti G., Wahl J.H., Beauchamp G.K., (2006) Immunogenetics.58(12):967-82.

[27] Knapp L.A., Robson J., Waterhouse J.S.,(2006) Am J Primatol.68(6):56884.

[28] Wright P.E., Dyson H.J., Lerner R.A., Riechmann L., Tsang P., (1990) Biochem Pharmacol. 40(1):83-8.

[29] Niemann H. $2^{\text {nd }}$ European Veterinary Immunology Workshop, Paris, September 4th-6th 2006, PS3-01, 46.

[30] Niewold T.A., Veldhuizen E.J.A., van der Meulen J., Haagsman H.P., Smits M.A., Hulst M.M. $2^{\text {nd }}$ European Veterinary Immunology Workshop, Paris, September 4th-6th 2006, PS302, 46.

[31] Flori L., Mariani V., Rogel-Gaillard C., Cochet M., Bidanel J.P., Chardon P., Lefèvre F. $2^{\text {nd }}$ European Veterinary Immunology Workshop, Paris, September 4th-6th 2006, PS3-03, 46.

[32] Bender B., Bodrogi L., Mayer B., Schneider Z., Baranyi M., Zhao Y., Hammarstrom L., Bosze Z., Kacskovics I. $2^{\text {nd }}$ European Veterinary Immunology Workshop, Paris, September 4th-6th 2006, PS3-05, 47.

[33] Rochelle E.S., Schook L.B. $2^{\text {nd }}$ European Veterinary Immunology Workshop, Paris, September 4th-6th 2006, PS307, 47.

[34] De Groot A.S. (2006) Drug Discov Today.11(5-6), 203-9.

[35] Simone Mocellin, Ena Wang, Monica Panelli, Pierluigi Pilati and Francesco M. (2004) Clinical Cancer Research, 10, 4597-4606. 